\title{
A statistical study of SUMER spectral images: events, turbulence, and intermittency
}

\author{
E. Buchlin ${ }^{1,2, \star}$, J.C. Vial ${ }^{1}$, and P. Lemaire ${ }^{1}$ \\ ${ }^{1}$ Institut d'Astrophysique Spatiale, CNRS - Université Paris-Sud, Bât. 121, 91405 Orsay Cedex, France \\ e-mail: e.buchlin@imperial.ac.uk \\ 2 Dipartimento di Astronomia e Scienza dello Spazio, Università di Firenze, Largo E. Fermi 2, 50125 Firenze, Italy \\ Received 26 October 2005 / Accepted 23 January 2006
}

ABSTRACT

\begin{abstract}
We analyze a series of full-Sun observations performed with the SoHO/SUMER instrument between March and October 1996. Some parameters (radiance, shift and width) of the S VI $93.3 \mathrm{~nm}, \mathrm{~S}$ VI $94.4 \mathrm{~nm}$, and Ly $\varepsilon$ line profiles were computed on board. Radiances and line-of-sight velocities in a large central region of the Sun are studied statistically: distributions of solar structures, field Fourier spectra and structure functions are obtained. The structures have distributions with power-law tails, the Fourier spectra of the radiance fields also display power laws, and the normalized structure functions of the radiance and velocity fields increase at small scales. These results support the idea of the existence of small scales, created by turbulence, and of intermittency of the observed fields. These properties may provide insight into the processes needed for heating the transition region, or, if confirmed in the corona, the corona itself. The difficulties encountered in this analysis, especially for the velocity data, underline the need for sensitive ultraviolet imaging spectrometers.
\end{abstract}

Key words. Sun: transition region - Sun: flares - turbulence

\section{Introduction}

The mechanism heating the solar corona to millions of degrees kelvin remains an open problem, but it is generally understood that a large part of the energy dissipation must occur at scales that are smaller than the structures that can be resolved by observations $(\approx 100 \mathrm{~km})$, perhaps as small as 10 to $100 \mathrm{~m}$ (the Kolmogorov turbulent cascade dissipation scale). One of the most successful approaches to fill this four-order-of-magnitude wide gap is to assume that the statistics obtained at observable scales are still valid at the smallest scales. The properties of the global system, from observable to non-observable scales, can then be investigated. This, for example, is the idea underlying Hudson's (1991) critical power-law slope of -2 for the distribution of flare energies.

\subsection{Statistics from previous observational studies}

Distributions of events. The measurement of the power-law slope for the lowest energy flares has been a major goal of coronal physics in the last decade. Aschwanden et al. (2000) summarized the distributions of event energies that were obtained at wavelengths from $\mathrm{X}$ rays to ultraviolet (UV), and for event energies covering eight orders of magnitude from $10^{17} \mathrm{~J}$ ("nanoflares") to $10^{25} \mathrm{~J}$ ("flares"). It seems, as stressed by Aschwanden, that these distributions can be combined to form a unique power-law distribution of slope $\approx-1.8$.

Spectra. Fourier spectra of the radiance fields in UV and X rays have also been obtained (e.g. Martens \& Gomez 1992; Gomez et al. 1993; Benz et al. 1997; Berghmans et al. 1998, from

* Present address: Space and Atmospheric Physics Department, Imperial College, London SW7 2BW, UK.
Yohkoh/SXT, NIXT and EIT). Most of them display power laws (with indices of -1.4 to -4 ), indicating the probable presence of turbulence.

Structure functions. Using transverse structure functions, Abramenko et al. (2002) have found that the photospheric magnetic field in active regions is intermittent (and intermittency is higher for high activity). Patsourakos \& Vial (2002) have also found intermittency in the time series of radiance (light curves) of the lines Ne VIII $77.04 \mathrm{~nm}$ and O IV $79.02 \mathrm{~nm}$. Intermittency has also been observed in situ in the solar wind velocity and magnetic field fluctuations (Burlaga 1991; Marsch \& Tu 1997).

\subsection{Aim of this study and selection of the data}

Almost all the statistics of UV observations cited above were done on radiance images obtained by pass-band filter imaging instruments. Here we want to obtain statistics of the coronal turbulence and subsequent heating, and this requires access to more direct signatures of the basic fields of MHD, namely the 3D magnetic and velocity fields. Some statistics of the photospheric magnetic field have been obtained (cf. Abramenko et al. 2002), but they are limited to the line-of-sight component. As for coronal magnetic fields, no measurement has been obtained over a whole solar area. Concerning the coronal velocity field, to our knowledge no statistical study has yet been done in the framework of turbulence, although velocity statistics are very important in the study of turbulence. To address this subject, we need to use spectroscopic data, and SoHO/SUMER (Wilhelm et al. $1995)$ is still one of the solar UV spectrographs with the best performance currently available. A spectrograph also allows us to obtain "purer" data in radiance, because the line radiance is obtained by line fitting which removes the continuum background. 
In order to perform statistics, we need large amounts of data, and also large fields with a good spatial resolution to compute Fourier spectra or structure functions over a wide range of scales (or frequencies). The resolution of SUMER $\left(\approx 1^{\prime \prime}\right)$ allows us to reach scales close to the smallest scales observable by current UV instruments. On the other hand, scales of the order of the size of the Sun can be reached by SUMER when rastering the whole image of the Sun, as has been done in the "full-Sun" observation program which is detailed in Sect. 2.1.

We choose to restrict ourselves to quiet regions of the Sun. We examine statistical results on the turbulent state of the corona and on the resulting events of energy dissipation that heat the whole corona, active and quiet, and there is no reason a priori to exclude specific features like the supergranulation pattern or active regions. On the other hand, the statistical weight of one active region may be too high compared to the total amount of data we have, and the characteristics of a particular active region may influence the overall statistics. For this reason, we exclude data containing active regions from our analysis (but we do not perform any particular treatment concerning supergranulation); an analogous study of active regions will be the subject of a subsequent work.

\section{The data}

\subsection{Observation program and data set}

We use data from a series of 36 observations of the full Sun, done in 1996 by one of us (PL). These data (Table 1) were obtained by the SUMER spectrograph aboard the SoHO satellite (Wilhelm et al. 1995), and taken from the SoHO archive at MEDOC ${ }^{1}$. At that time, most of the Sun was quiet, and active regions were rare. In each observation of this program, 8 rasters in the eastwest direction were done with slit number $2\left(1^{\prime \prime} \times 300^{\prime \prime}\right)$, and they were accumulated in the north-south direction to obtain full-Sun images. The exposure time was $3 \mathrm{~s}$ and the slit moved (in most cases) by 4 elementary steps $\left(1.52^{\prime \prime}\right)$ in the east-west direction.

Spectra were obtained with detector A of SUMER, but contrary to other observations with this instrument, these spectra (or parts of spectra) were not sent to the ground: only 5 parameters of 3 spectral lines were transmitted for each position on the Sun (i.e. position of the slit and pixel along the slit). These parameters were computed on board by SUMER. During this process, some information was lost, but this allowed us to get spectroscopic maps of the whole Sun at a high resolution $\left(1^{\prime \prime} \times 1.5^{\prime \prime}\right)$ with low telemetry use $\left(8 \times 10^{7}\right.$ bits $)$ and quickly (within $\left.9 \mathrm{~h}\right)$.

The following parameters (or "moments") of spectral line profiles were obtained ${ }^{2}$ :

- (1) peak spectral radiance, (2) Doppler shift and (3) width of the line S VI $93.3 \mathrm{~nm}$ (transition $2 \mathrm{p}^{6} 3 \mathrm{~s}^{2} \mathrm{~S}_{1 / 2}-2 \mathrm{p}^{6} 3 \mathrm{p}^{2} \mathrm{P}_{3 / 2}$ at $93.340 \mathrm{~nm}$, of mean peak of spectral radiance $\left.0.57 \mathrm{~W} \mathrm{~m}^{-2} \mathrm{sr}^{-1} \mathrm{~nm}^{-1}\right)$;

- (4) line radiance (integrated spectral radiance) of the line Ly $\varepsilon$ (transition $1 \mathrm{~s}^{2} \mathrm{~S}_{1 / 2}-6 \mathrm{p}^{2} \mathrm{P}_{3 / 2}$ at $93.780 \mathrm{~nm}$, of mean peak of spectral radiance $1.07 \mathrm{~W} \mathrm{~m}^{-2} \mathrm{sr}^{-1} \mathrm{~nm}^{-1}$ );

- (5) line radiance of the line S VI $94.4 \mathrm{~nm}$ (transition $2 p^{6} 3 s^{2} S_{1 / 2}-2 p^{6} 3 p^{2} P_{1 / 2}$ at $94.455 n m$, of mean peak of spectral radiance $0.29 \mathrm{~W} \mathrm{~m}^{-2} \mathrm{sr}^{-1} \mathrm{~nm}^{-1}$ ).

\footnotetext{
1 The MEDOC archive is publicly available from http://www . medoc-ias.u-psud. fr

2 Spectroscopic data and the mean peak of spectral radiances (given for the quiet Sun) are taken from Curdt et al. (2001).
}

Table 1. List of the observations of the full Sun done in 1996. The 4digit name corresponds to the date of the start of the observation (which may end on the following day). Notes: AR, active region near the center of the field of view; P1, some pointing problems, or missing data, outside of the center of the field of view; P2, these problems may affect the center; P3, important problems of pointing or missing data; 1 , the raster step in the east-west direction is $1.14^{\prime \prime}$ ( 3 elementary steps) instead of $1.52^{\prime \prime}$ (4 elementary steps).

\begin{tabular}{|c|c|c|c|c|}
\hline Name & Date & Start & End & Notes \\
\hline 0401 & $\begin{array}{l}1996 / 04 / 01 \\
\end{array}$ & $12: 30: 43$ & $17: 20: 57$ & 1 \\
\hline 0407 & $1996 / 04 / 07$ & $20: 24: 54$ & $04: 56: 18$ & \\
\hline 0414 & $1996 / 04 / 14$ & 01:02:49 & $09: 34: 15$ & \\
\hline 0418 & $1996 / 04 / 18$ & $20: 11: 39$ & 04:43:05 & \\
\hline 0424 & $1996 / 04 / 24$ & $11: 08: 45$ & $19: 40: 12$ & \\
\hline 0429 & $1996 / 04 / 29$ & $01: 26: 38$ & 09:58:03 & \\
\hline 0504 & $1996 / 05 / 04$ & 07:43:09 & $16: 14: 36$ & \\
\hline 0508 & $1996 / 05 / 08$ & 05:09:03 & $13: 40: 28$ & \\
\hline 0512 & $1996 / 05 / 12$ & 23:02:03 & $07: 33: 28$ & AR \\
\hline 0517 & $1996 / 05 / 17$ & $00: 47: 02$ & $08: 32: 43$ & P1 \\
\hline 0524 & $1996 / 05 / 24$ & $08: 57: 22$ & $17: 28: 48$ & P1 \\
\hline 0528 & $1996 / 05 / 28$ & $12: 39: 04$ & $21: 10: 29$ & P1 \\
\hline 0603 & $1996 / 06 / 03$ & 21:13:56 & $05: 45: 21$ & P1 \\
\hline 0608 & $1996 / 06 / 08$ & $21: 10: 31$ & $05: 41: 56$ & P2 \\
\hline 0612 & $1996 / 06 / 12$ & $16: 12: 47$ & $00: 44: 13$ & P1 \\
\hline 0616 & $1996 / 06 / 16$ & $22: 41: 43$ & $07: 13: 10$ & \\
\hline 0621 & $1996 / 06 / 21$ & $22: 48: 43$ & $07: 20: 10$ & P3 \\
\hline 0701 & $1996 / 07 / 01$ & $19: 42: 26$ & $04: 13: 48$ & P3 \\
\hline 0706 & 1996/07/06 & $22: 09: 36$ & $06: 41: 02$ & AR P2 \\
\hline 0711 & $1996 / 07 / 11$ & $17: 44: 09$ & $02: 15: 35$ & \\
\hline 0716 & $1996 / 07 / 16$ & $19: 13: 27$ & $03: 44: 49$ & P2 \\
\hline 0721 & $1996 / 07 / 21$ & 18:38:19 & 03:09:48 & \\
\hline 0726 & $1996 / 07 / 26$ & 00:19:04 & $08: 50: 28$ & \\
\hline 0801 & 1996/08/01 & $21: 07: 56$ & $05: 39: 21$ & AR \\
\hline 0806 & 1996/08/06 & $19: 14: 26$ & 00:04:04 & 1 \\
\hline 0811 & $1996 / 08 / 11$ & $16: 19: 29$ & $00: 50: 53$ & AR \\
\hline 0814 & $1996 / 08 / 14$ & 06:07:09 & $14: 05: 05$ & P3 \\
\hline 0816 & $1996 / 08 / 16$ & $20: 28: 22$ & $04: 59: 45$ & AR P2 \\
\hline 0821 & $1996 / 08 / 21$ & $14: 41: 18$ & $23: 12: 42$ & $\mathrm{P} 2$ \\
\hline 0828 & $1996 / 08 / 28$ & 09:52:13 & $12: 53: 48$ & AR P3 \\
\hline 0902 & $1996 / 09 / 02$ & 00:11:00 & $08: 42: 22$ & P3 \\
\hline 0906 & $1996 / 09 / 06$ & $04: 23: 15$ & $12: 54: 41$ & \\
\hline 0913 & $1996 / 09 / 13$ & $21: 36: 32$ & 06:07:58 & P3 \\
\hline 0924 & $1996 / 09 / 24$ & $15: 22: 43$ & $23: 53: 26$ & $\mathrm{AR}$ \\
\hline 0930 & $1996 / 09 / 30$ & $22: 02: 21$ & $06: 33: 47$ & \\
\hline 1005 & $1996 / 10 / 05$ & $04: 42: 48$ & $13: 14: 14$ & \\
\hline
\end{tabular}

Hereafter the moments will sometimes be referred to by their numbers.

The Ly $\varepsilon$ line is emitted at a formation temperature of around $10000 \mathrm{~K}$ in the high chromosphere, and the S VI lines are emitted around $200000 \mathrm{~K}$ in the transition region between the chromosphere and the corona. The width ("moment" 2 ) is computed (on board) as the width of the interval where the line profile is higher than half its peak values, and the Doppler shift (moment 1) is the position of the center of this interval. The data are then compressed aboard SoHO (to one byte per moment per pixel), and decompressed on the ground before being analyzed (see details of compression in Appendix A).

In addition, some full spectra were recorded so as to check for the positions of the spectral windows (used for the on board computations) and for the global reliability of the computations. One of these is shown in Fig. 1. No specific problem was detected. The design of SUMER, in particular the absence of cosmic rays impacts on the detector, makes the computation of line parameters aboard quite reliable, notwithstanding the noise problem (see below in Sect. 2.3). 

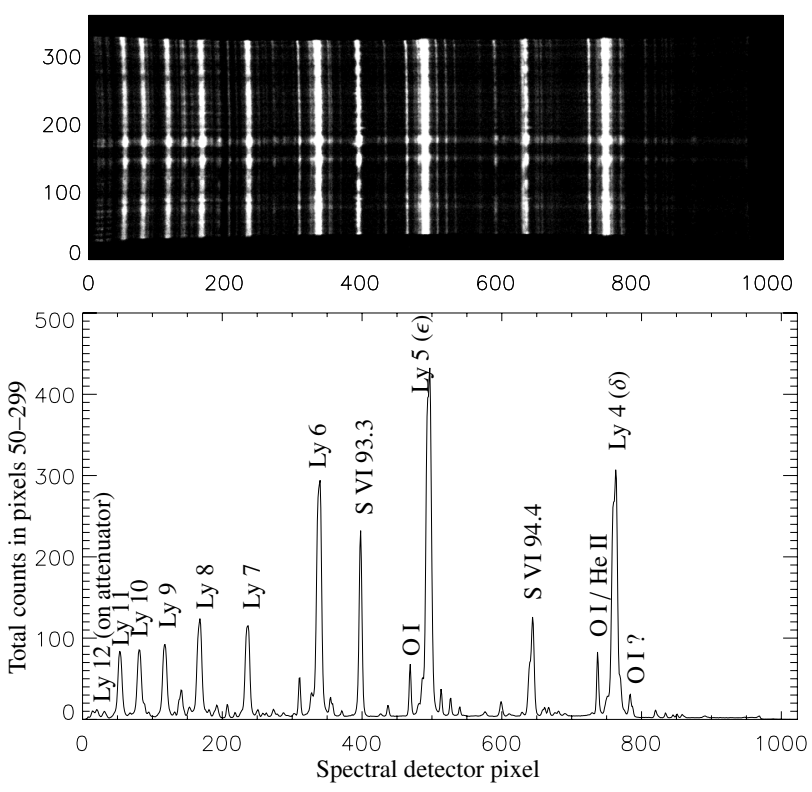

Fig. 1. Top: context spectrum (full detector A) taken on 4 May 1996 at $7 \mathrm{~h} 32 \mathrm{~min}$ UT. Bottom: total counts along slit between pixels 50 and 299. The S VI lines and the Lyman series of hydrogen (from Ly 4 to Ly 12) can be seen.

\subsection{Correction of the data}

As all the data do not come with line profiles, the usual procedures of dark current removal and flatfield correction cannot be applied. Thus we have to assume that the variations along the slit of averages of data for different dates and positions result from systematic instrumental effects and not from solar structures (actually, large structures like active regions were excluded from the computation of the averages). These averages, shown in Fig. 2, are then subtracted from the raw data (for Doppler shift) or the raw data is divided by the average (for radiances) to get corrected data. For convenience, the units of the resulting data are hereafter called "data units" and denoted du.

\subsection{Data values and noise}

The context spectra (Fig. 1), which were taken with an exposure time of $300 \mathrm{~s}$, show most line profiles with a few hundreds of total counts at peak, whereas the full-Sun images were taken with a $3 \mathrm{~s}$ exposure time. The counts on the detector during the fullSun observations are therefore low, which is confirmed by the distributions of the values of radiance moments (1), (4) and (5) shown in Fig. 5d, and noise is an issue. Monte Carlo simulations were performed to estimate the influence of counting statistics on the data: we started from Gaussian profiles with background, whose amplitudes were chosen according to the profiles of the context spectra $\left(0.1\right.$ counts pixel ${ }^{-1}$ for the background, and

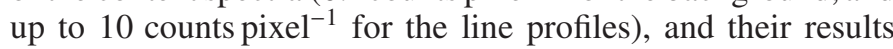
(noise as a function of the line amplitude, for each kind of moment) are shown in Fig. 3. The detector noise, of the order of $10^{-4}$ counts s $^{-1}$ pixel $^{-1}$, is not taken into account. Taking an amplitude of 4 counts pixel ${ }^{-1}$ as an example, the noise (standard deviation of the line parameters computed from the Monte Carlo simulations) is:

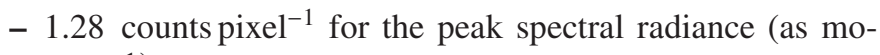
ment 1);

- 1.64 pixels for the line shift (as moment 2);
(1) S VI $93.3 \mathrm{~nm}$ peak spectral radiance (counts)

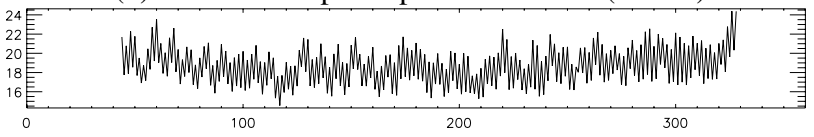

(2) S VI $93.3 \mathrm{~nm}$ line shift (pixels)

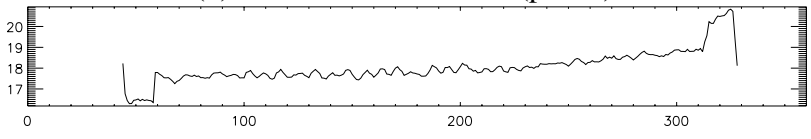

(3) S VI $93.3 \mathrm{~nm}$ line width (pixels)

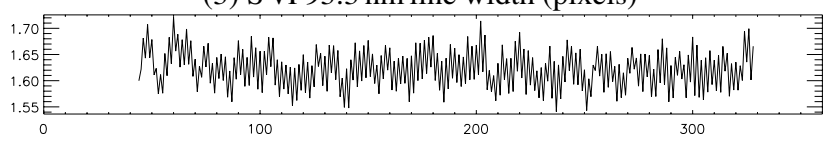

(4) Ly $\varepsilon$ radiance (counts)

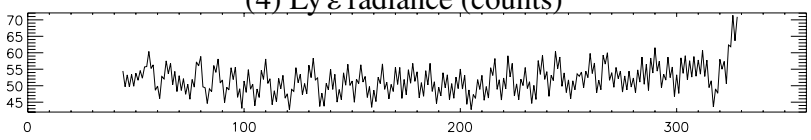

(5) S VI 94.4 nm intensity

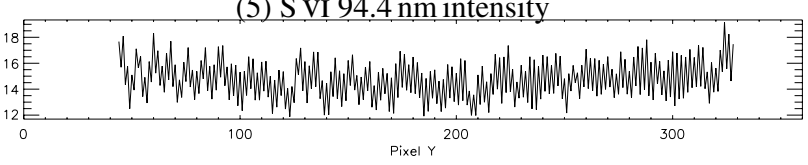

Fig. 2. Average profiles along the slit over all images (excluding specific structures), for each of the 5 moments, in counts or pixels. In particular, the well-known odd-even pattern of the detector is clearly visible. Note that these cuts represent averages around disk center only.
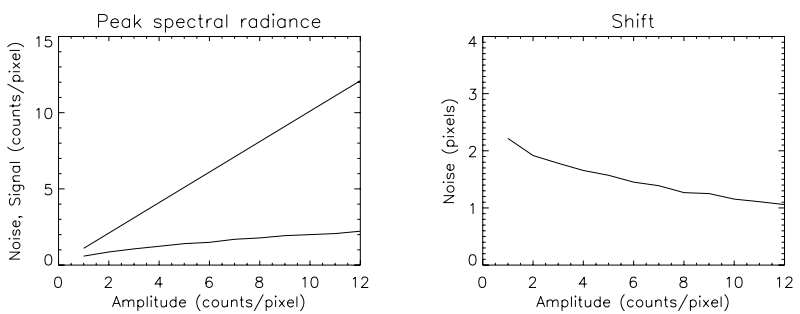

Width
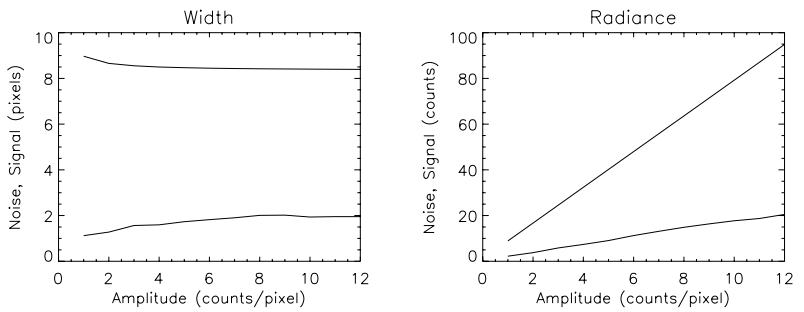

Fig. 3. Noise (lower curves) and signal (upper curves, when relevant) in each pixel as a function of the amplitude of the Gaussian profile used for the Monte Carlo simulations, for peak spectral radiance, line shift, line width, and line radiance.

- 1.77 pixels for the line width (as moment 3);

- 8.4 counts for the line radiance (as moments 4 and 5).

These values, extracted from the plots of Fig. 3, are consistent with the sensitivity predicted during the mission preparation (Wilhelm et al. 1989).

Among the radiance and line shift data we use, the most acute noise problem comes from moment 2 (Doppler shift of $\mathrm{S}$ VI $93.3 \mathrm{~nm}$ ): as can be seen in Fig. 4, most of the data are contained in the error bars coming from a $1-\sigma$ evaluation of the noise from the Monte-Carlo simulations, thus noise can account for most of the dispersion of the data in moment (2). Furthermore this moment is saturated due to the limited width of the spectral 


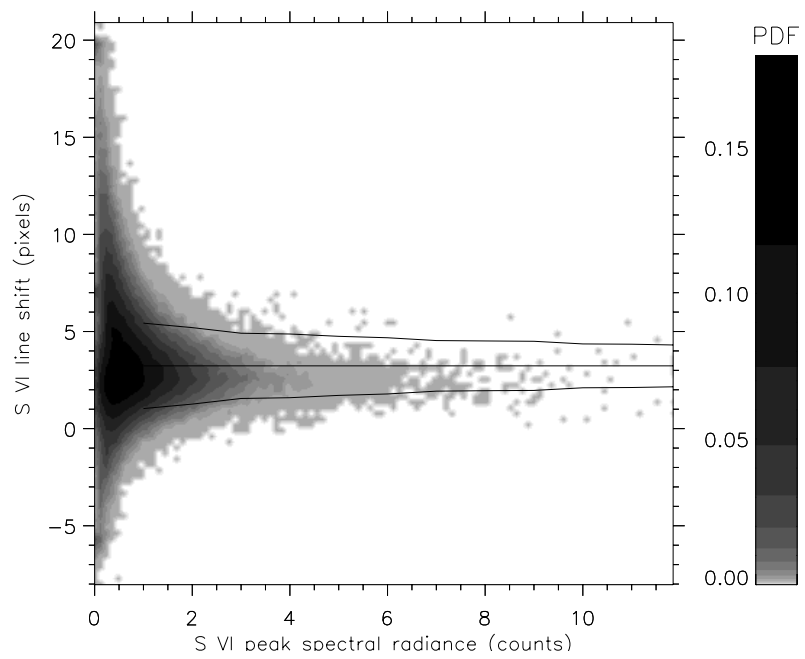

Fig. 4. Joint probability distribution function (PDF) of moments 1 (S VI $93.3 \mathrm{~nm}$ peak spectral radiance) and 2 (S VI $93.3 \mathrm{~nm}$ line shift), in data units (counts and pixels). The horizontal line superimposed on this plot is the median of moment (2), and the other curves are the median plus or minus the standard deviation of the noise computed by the Monte Carlo simulations (Fig. 3), as a function of moment (1), assuming that the noise in moment (1) is low.

window used to compute the line shift and due to the compression algorithms (Appendix A), and this saturation is still visible in the wings of the distribution of the corrected moment (as seen in Fig. 5d.2); however, this concerns only $0.5 \%$ of the pixels and the effect on the statistics is weak.

\subsection{Construction of full-Sun images}

The 8 rasters for each observation are put together first by using the pointing coordinates from the headers of the data files then by a further adjustment so that the limbs on neighboring rasters fit exactly. Figure 5a shows the resulting images for observation 0721. However, the boundaries between rasters can still be seen, mainly because the observation times of adjacent pixels in different scans can be separated by up to $2 \mathrm{~h}$, and during this time the Sun has rotated by $1.1^{\circ}$ in longitude, or $19^{\prime \prime}$ at disk center as seen by SoHO. For this reason, when the statistics imply spatial information, we work with data coming from only one raster at a time. This fact does not reduce the interest of using these full-Sun images to do statistics, as we still have data with a large number of pixels produced in a short time.

\subsection{Profiles of the spectral images along the equator}

We produce averaged profiles of the corrected moments along the equator of the Sun, in a 200"-wide band on either side (note that here and hereafter we approximate the Sun by a sphere and the projection of the solar equator with the solar disk diameter which is parallel to the $X$ axis). To do this, we need to (1) take into account the variation of the apparent size of the Sun (due to the eccentricity of SoHO's orbit around the Sun), which can be seen in Fig. 6: the solar $X$ coordinates of the profiles are normalized to a common solar diameter before computing the average of the profiles; (2) take into account the curvature of the limb along the width of the band used to compute the average profiles. The resulting profiles, shown in Fig. 5b, present interesting features, like strong limb brightenings in the S VI lines (moments 1 and 5), which can also be seen in the images of Fig. 5a. The average profiles of these radiances are consistent with the theoretical $1 / \mu$ limb brightening of optically thin lines (with $\mu=$ $\cos \alpha$ and $\alpha$ the angle between the normal to the solar surface and the line of sight). The fit for moment 1 (S VI $93.3 \mathrm{~nm}$ spectral radiance) gives $(0.14 \pm 0.05)+(0.75 \pm 0.03) / \mu$ with $\chi^{2}=5.7 \times 10^{-3}$ and is shown in Fig. 7. A limb brightening in the Ly $\varepsilon$ radiance (moment 4), although weaker because this line is optically thicker than the S VIlines, has also been measured and can be seen in Figs. 5a, b.

The S VI $93.3 \mathrm{~nm}$ line shift (moment 2), also shown in Fig. 5b, presents a curvature that can be interpreted as follows. Let $\theta$ and $\varphi$ be respectively the heliographic latitude and longitude, with the origin on the line of sight towards the observer. Assuming that the Sun is a sphere of radius $R$, rotating at an angular velocity $\Omega(\theta)$ around a north-south axis perpendicular to the line of $\operatorname{sight}^{3}$, and assuming that matter flows down at a radial velocity $u(\theta, \varphi)$, the observed velocity, projected on the line of sight, with positive values for downflows, is:

$$
\begin{aligned}
v_{\mathrm{los}} & =\Omega(\theta) \cdot R \cos \theta \cdot \sin \varphi+u(\theta, \varphi) \cdot \cos \theta \cdot \cos \varphi \\
& =\Omega(\theta) \cdot x+u(\theta, \varphi) \cdot \sqrt{1-\left(x^{2}+y^{2}\right) / R^{2}}
\end{aligned}
$$

where $x=R \cos \theta \cdot \sin \varphi$ and $y=R \sin \theta$ are the heliocentric coordinates. The fit of the profile of moment (2) by this function (Fig. 8) gives $\Omega=(5 \pm 5) \times 10^{-5} \mathrm{du} /{ }^{\prime \prime}$ and $u=1.8 \pm 0.1 \mathrm{du}$ with $\chi^{2}=7.9 \times 10^{-3}$, where du is the data unit for moment (2). Note that a null velocity corresponds to $-1.6 \pm 0.1 \mathrm{du}$.

On the other hand, the unit of line shift given by the decompression routines is one pixel on the detector in the direction of spectral dispersion, i.e. $0.0044 \mathrm{~nm}$ at a wavelength of $\lambda=94 \mathrm{~nm}$, or a $14 \mathrm{~km} \mathrm{~s}^{-1}$ downflow. We would thus expect that $\Omega=15 \times 10^{-5} \mathrm{du} /{ }^{\prime \prime}$. There is a disagreement with the value of $\Omega$ obtained previously, indicating that the fit of solar rotation from average equatorial velocity profiles is perhaps not a good way to determine the velocity calibration in the case of these observations. The origin of this problem may be the low signal (see Sect. 2.3), which makes it difficult to see the effects of solar rotation (expected to be $\pm 2 \mathrm{~km} \mathrm{~s}^{-1}$ on the limb). We retain the value $14 \mathrm{~km} \mathrm{~s}^{-1}$ for the velocity unit, which seems to be the most reliable and which gives physical values for $u$ which are more consistent with previous observations in the same line (Brekke et al. 1997; Chae et al. 1998; Peter \& Judge 1999).

The S VI $93.3 \mathrm{~nm}$ line width (moment 3 ) is also larger near the limb. This increase is easily explained by an increase at the limb (due to projection) of the optical depth of this optically thin line: the velocity field of the matter emitting in this line is averaged over a longer line-of-sight near the limb than at disk center.

\section{Statistical analysis}

\subsection{Statistics of structures}

Bright structures visible in images can be identified with energy dissipation events. Here we use the same method as in Aletti et al. (2000) to define structures: one structure is a set of pixels whose radiance is above a threshold $L_{\mathrm{thr}}$, i.e. a maximal connex part (cluster) of the set of image pixels with

\footnotetext{
3 We consider that the heliographic latitude $B_{0}$ of the disk center is 0 , i.e. that $\mathrm{SoHO}$ is in the equatorial plane; this is an approximation due to the angle between this plane and the ecliptic $\left(7.23^{\circ}\right)$, and because of the excursions of SoHO's orbit outside the ecliptic. But it would only add a second-order contribution to the restriction of Eq. (1) to the equator $(\theta=0)$.
} 

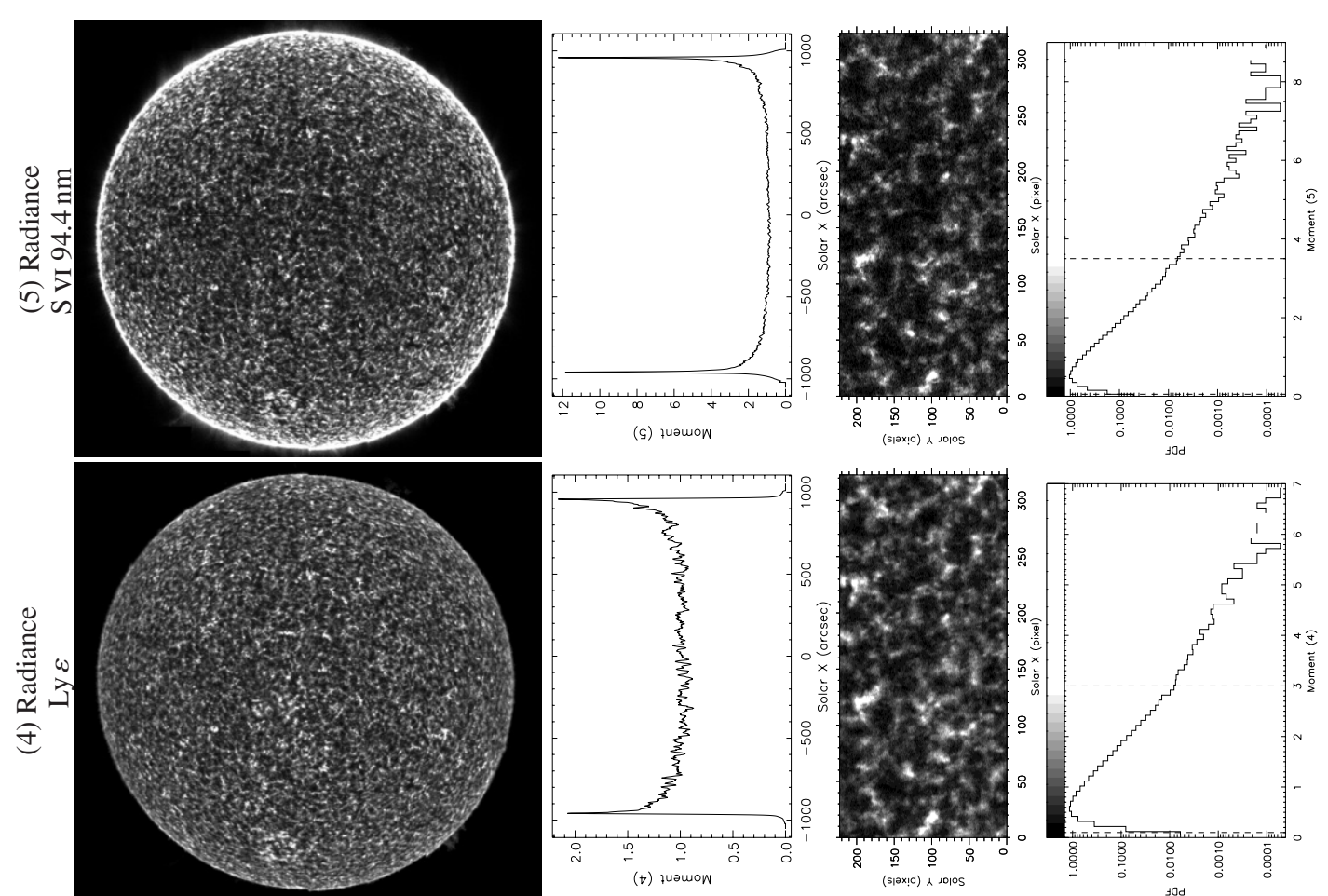

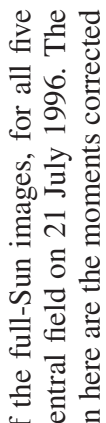

पे

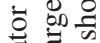

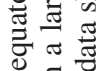
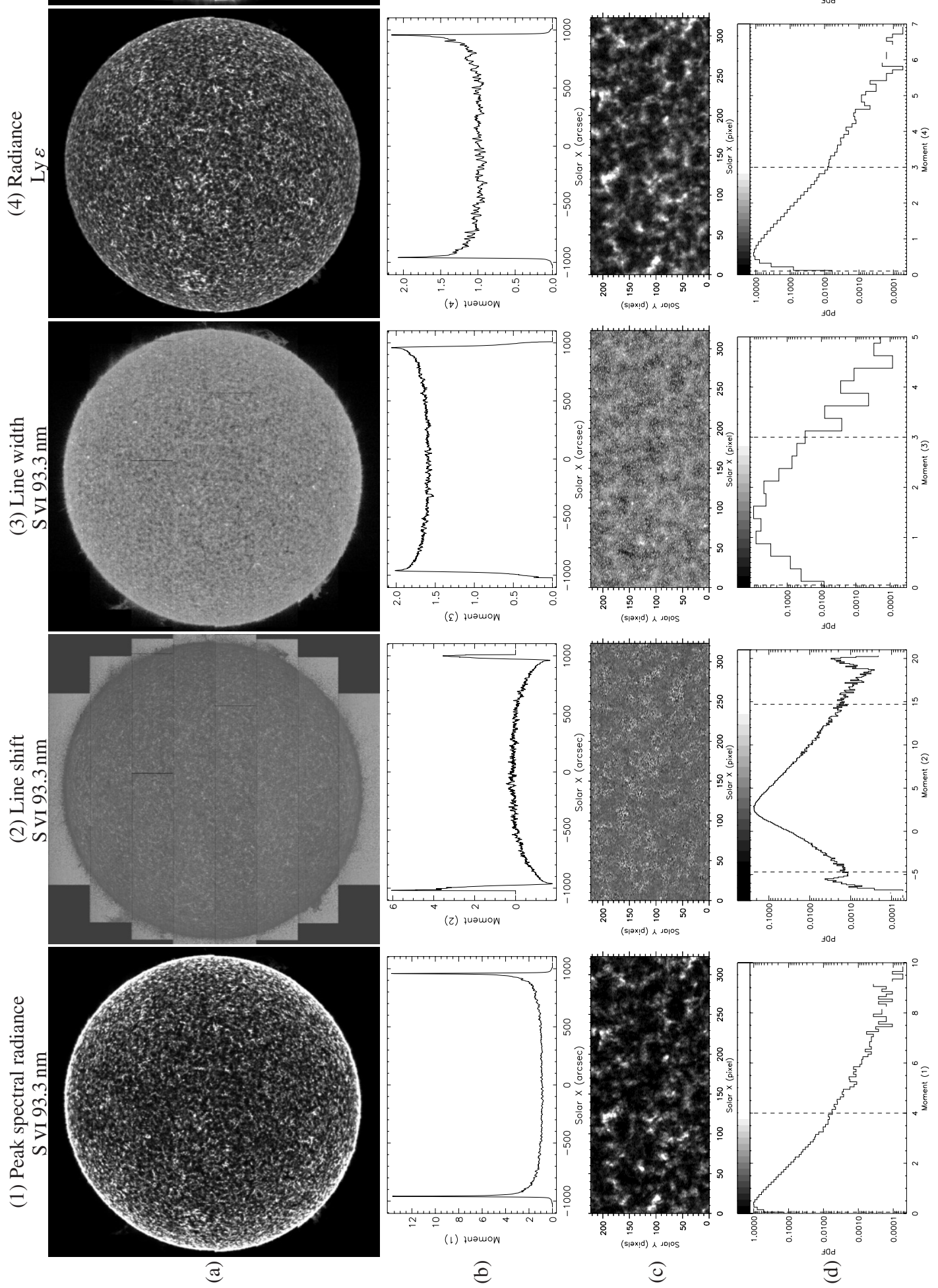

Ð

요

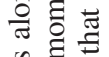

苞导

:

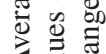

을.

원유.

क है

퓸

范

동

$\sum^{2}$

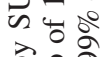

웜

氙

है चี

응

氙

药

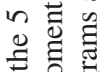

。․

○。

ลㅋㅇㅇ

홍

ㄱํㅇำ

드는

छ․․․

ज咣

छ 응

휸

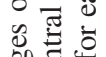

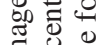

ఏ త్ర్లే

氜 就

플

至芯芯

워워

ส

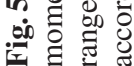



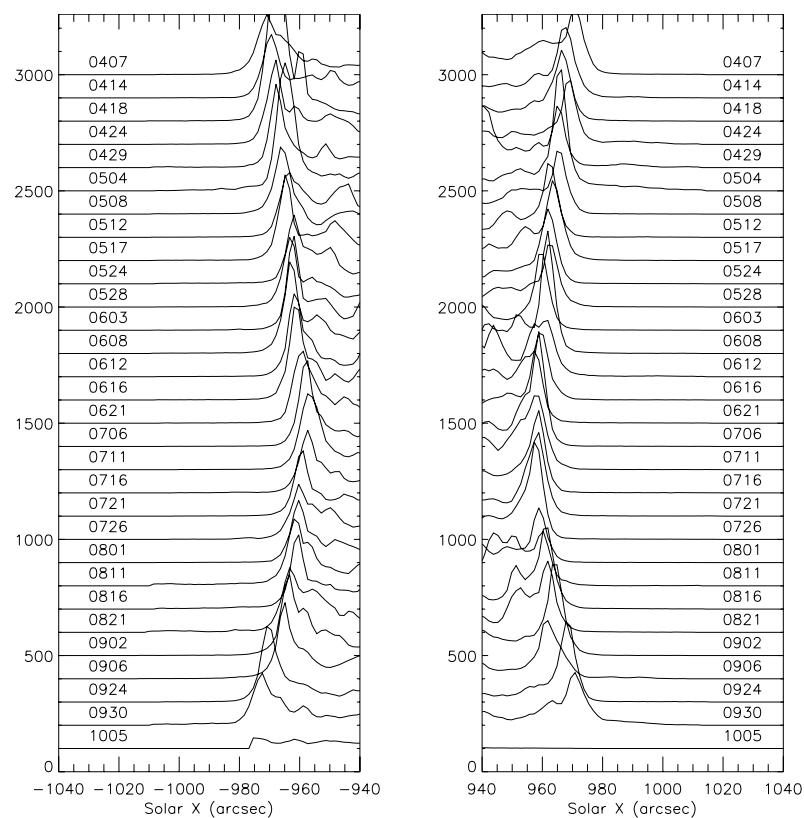

Fig. 6. Profiles of S VI $93.3 \mathrm{~nm}$ radiance in a 100-pixel-wide band around the equator, as a function of solar $X$, for all observation dates. The units of radiance are data units, and each profile is shifted by 100 units from the previous one.

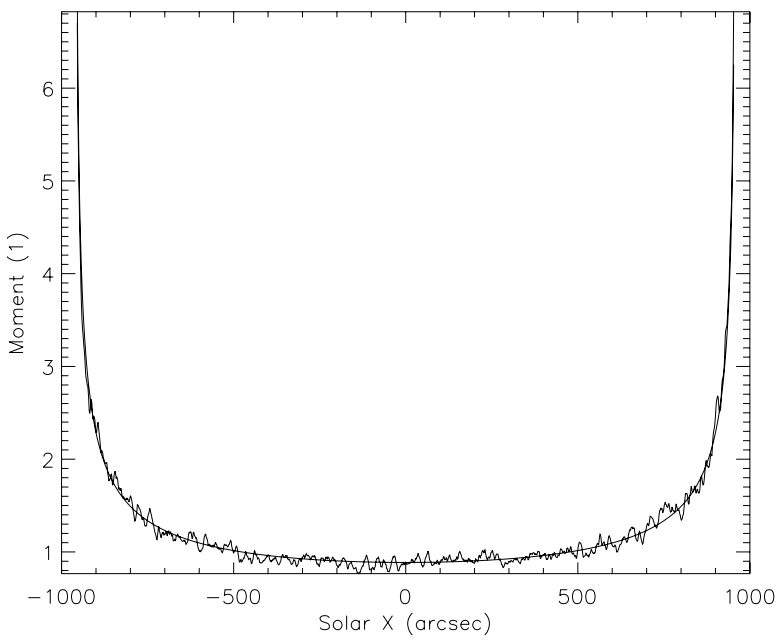

Fig. 7. Average profile of moment (1) (S VI $93.3 \mathrm{~nm}$ peak spectral radiance, in data units) along the equator, fitted to the $1 / \mu$ theoretical function. Pixels at the limb and outside of the limb, where the optically thin plan-parallel approximation is not valid anymore, have been excluded from the plot.

radiance above the threshold. Such structures, found using moment (1) (S VI $93.3 \mathrm{~nm}$ spectral radiance) on 21 July 1996 and with a threshold $L_{\mathrm{thr}}$ set to the average radiance plus one standard deviation, are outlined in Fig. 9 (top); 2313 of them were found in a central area of $700 \times 270$ pixels. Since the time difference between successive images is too long, we cannot use the time information as in Parnell \& Jupp (2000).

Let $P_{k}=\left\{p_{j, k}, j \in J_{k}\right\}$ be the set of pixels in a structure labeled $k\left(J_{k}\right.$ is the set of the indices $j$ of these pixels); the radiance (e.g. moment 1 ) of the pixel $p_{j, k}$ is called $L_{j, k}$. The intensity $I_{k}$ of the event of energy dissipation corresponding to the structure labeled $k$ is then defined as the integrated radiance on the structure, i.e. $I_{k}=\sum_{j \in J_{k}} L_{j, k}$. For the central field of 21 July 1996,

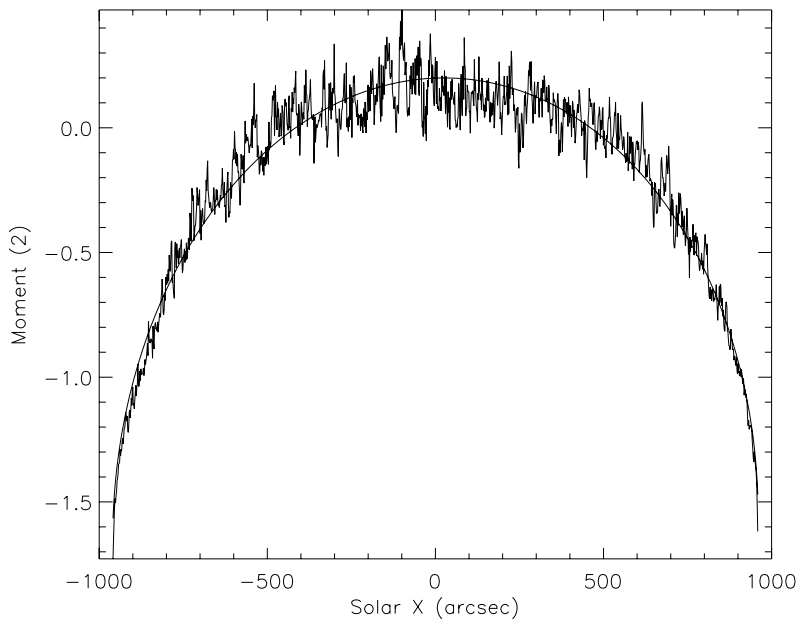

Fig. 8. Average profile along the equator of moment (2) (line shift of $\mathrm{S}$ VI $93.3 \mathrm{~nm}$, in data units), adjusted to the theoretical profile given by Eq. (1).

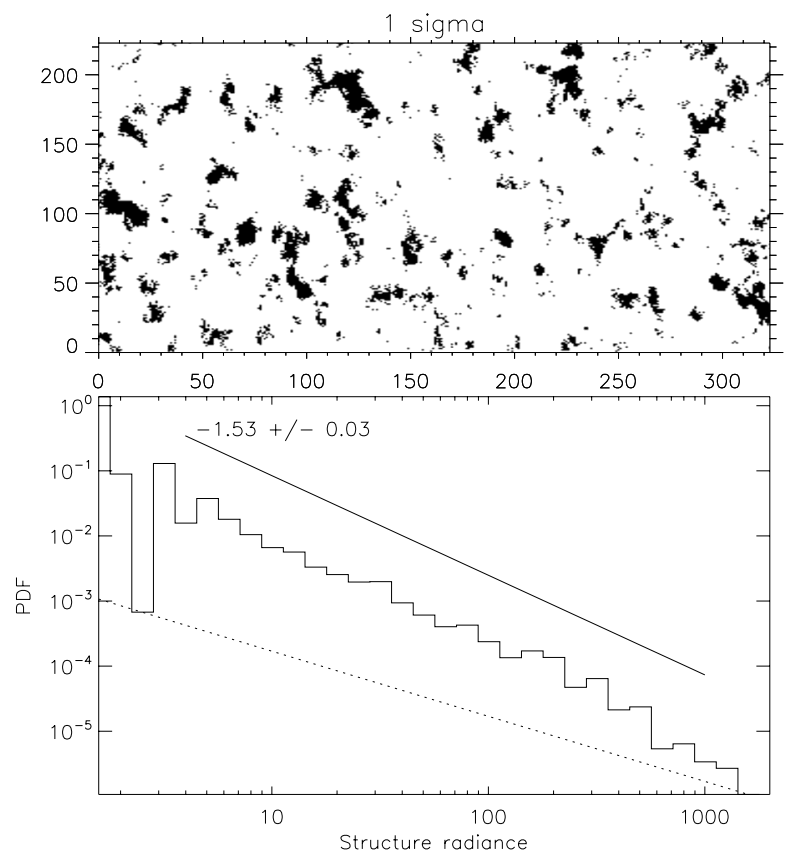

Fig. 9. Top: structures (black clusters) determined by a spectral radiance threshold of S VI $93.3 \mathrm{~nm}$ (average plus one standard deviation), on a part of the central field, on 21 July 1996. Bottom: probability distribution function (PDF) of the spectral radiance integrated over these structures, and its fit to a power law. The dotted line represents one event per variable-width histogram bar and is shown as an indication of the statistical noise coming from the construction of the histogram.

we obtain a power-law distribution ${ }^{4}$ of index $-1.53 \pm 0.03$ over more than two decades, as can be seen on the bottom of Fig. 9 . This analysis is repeated on the 25 best images, and we obtain 40920 structures, whose intensity is distributed as a power law of index $-1.57 \pm 0.01$ (Fig. 10). Compared to the distribution for 21 July 1996, this distribution is extended towards high intensities because of rare, but intense, events appearing at some other dates. The results for the other radiance moments are similar and are summarized in Table 2. The error bars are the uncertainties

4 The probability distribution functions (PDF) are obtained by building an histogram and then dividing the height of the bars by the total number of elements and by the width of the current bar. 


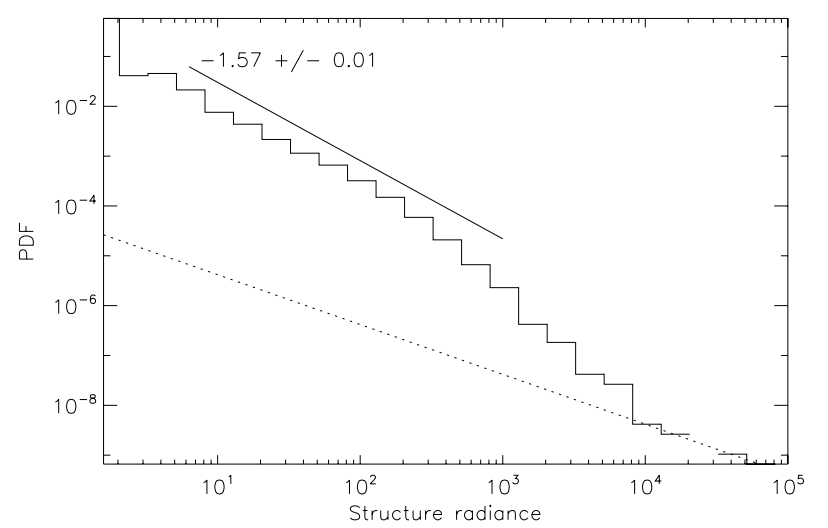

Fig. 10. Distribution of the $S$ VI $93.3 \mathrm{~nm}$ radiance integrated over structures defined as in Fig. 9 and over the 25 best images, and its fit to a power law. The dotted line represents one event per variable-width histogram bar.

Table 2. Slopes of power-law distributions of structures found in the maps of radiance moments ( $\mathrm{S}$ VI $93.3 \mathrm{~nm}$ spectral radiance, Ly $\varepsilon$ radiance and $\mathrm{S}$ VI $94.4 \mathrm{~nm}$ radiance). Note that the given uncertainties do not take into account those coming from the choice of the fitting range (see text).

\begin{tabular}{rlll}
\hline \hline Obs. & Moment (1) & Moment (4) & Moment (5) \\
time (s) & S VI 93.3 nm & Ly $\varepsilon$ & S VI 94.4 nm \\
\hline On 0721 & $-1.53 \pm 0.03$ & $-1.46 \pm 0.03$ & $-1.67 \pm 0.03$ \\
On average & $-1.57 \pm 0.01$ & $-1.44 \pm 0.01$ & $-1.74 \pm 0.01$ \\
\hline
\end{tabular}

on the results of the linear fit of the histograms in logarithmic scale, taking into account Poisson statistics in the counts of events in each histogram bar. However, they do not take into account the uncertainties coming from the selection of the fitting range, which could be evaluated to 0.1 approximately.

These slopes are steeper than the slope -1.2 found with a similar method by Aletti et al. (2000) on SoHO/EIT images. On the other hand, they are less steep than the slopes of the distributions of event energies found by Aschwanden et al. (2000) or Parnell \& Jupp (2000) for example, who use a different method: they use time information (like time clustering to find events), and they use some hypotheses to derive event energies from their radiance data. This derivation is highly non-trivial, as it depends on the relation between temperature and thermal energy, on the relation between the area and thickness of the emitting structures, on temperature and density diagnostics from observations, on the filling factor, etc. Here we have chosen to remain close to the observable variables, so as to get results independent of the assumptions needed to derive event energies. However, note that the integrated radiance is not the event energy, and, as it is not computed over the whole spectrum, it is not even the radiative loss during the event.

\subsection{Fourier spectra}

The omnidirectional Fourier spectrum $P(k)$ of a field is obtained by computing the integral of the $2 \mathrm{D}$ Fourier power spectrum $P_{2 \mathrm{D}}\left(k_{x}, k_{y}\right)$ of the field (which takes into account the different resolutions in the $x$ and $y$ directions, and using a cosine apodization function in both directions) over a ring of radius $k$ :

$P(k) \mathrm{d} k=k \mathrm{~d} k \int_{0}^{2 \pi} \mathrm{d} \theta P_{2 \mathrm{D}}(k \cos \theta, k \sin \theta)$.
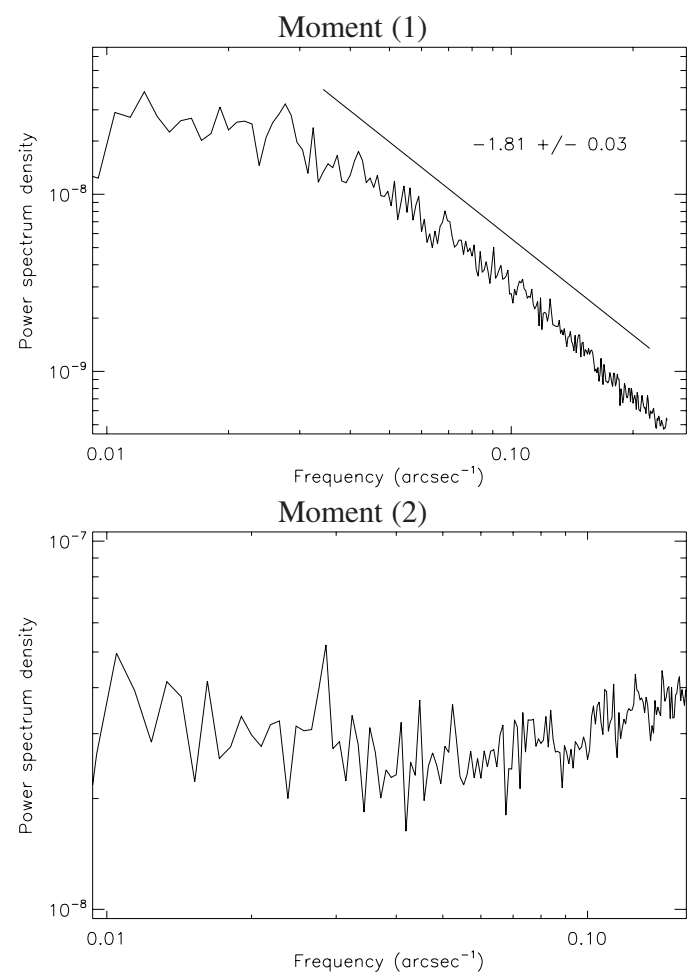

Fig. 11. Spatial Fourier spectra of moments 1 (S VI $93.3 \mathrm{~nm}$ spectral radiance) and 2 (S VI $93.3 \mathrm{~nm}$ line shift), on a central field of the Sun, on 21 July 1996.

In practice, this is done by computing the average of $P_{2 \mathrm{D}}$ over the grid points in 2D Fourier space that fall in the range $[k-$ $\delta k / 2, k+\delta k / 2]$ and by multiplying the result by $2 \pi k$.

$\mathrm{S}$ VI $93.3 \mathrm{~nm}$ spectral radiance. Figure 11.1 shows the spatial Fourier spectrum of the field of moment 1 (S VI $93.3 \mathrm{~nm}$ spectral radiance) as a function of the spatial frequency $f=k / 2 \pi$. This spectrum is fitted to a power law of index $-1.81 \pm 0.03$ for scales lower than $25 \mathrm{Mm}$ (corresponding to a $0.03^{\prime \prime-1}$ spatial frequency), where a break occurs. This scale is of the order of the scale of the supergranulation, and it is the same scale as the break in the Yohkoh/SXT X-ray radiance Fourier spectrum obtained by Benz et al. (1997), although the slopes of both parts of the spectrum are different there than in the case of our observations.

S VI $93.3 \mathrm{~nm}$ line shift. The power-law tail of the spectrum of the radiance field indicates that small scales exist, and they may be produced by turbulence. However, the direct measurement of the spectrum of the line-of-sight velocity (or line shift of S VI $93.3 \mathrm{~nm}$ ) - a physical parameter easier to compare to spectra predicted by theories of turbulence - does not yield satisfying results: these spectra are flat, as can be seen from Fig. 11.2 for 21 July 1996, implying that the noise in the maps of this parameter (moment 2) cannot be ignored.

\subsection{Structure functions}

For a scalar field $a(\boldsymbol{x})$, we define the increments $\delta_{\ell} a(\boldsymbol{x}) \equiv$ $a(\boldsymbol{x}+\boldsymbol{\ell})-a(\boldsymbol{x})$. The structure function $S^{q}(\ell)$ of index $q$ is the $q$ th moment of the increments $\delta_{\ell} a$ of the field at scale $\ell$, i.e.:

$S^{q}(\ell) \equiv\left\langle\left|\delta_{\ell} a(\boldsymbol{x})\right|^{q}\right\rangle_{\boldsymbol{x},\|\ell\|=\ell}$ 


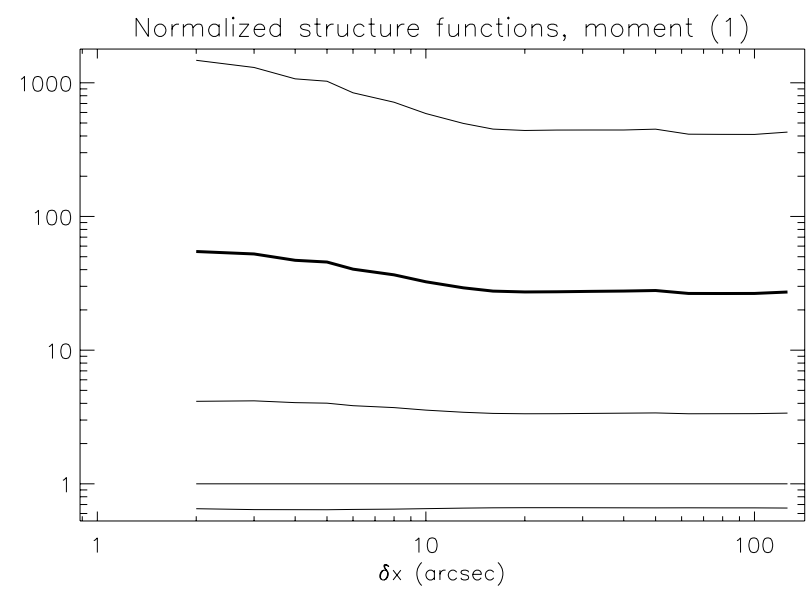

Fig. 12. Normalized structure functions of index 1 to 5 (from bottom to top) for the field of moment (1) (S VI $93.3 \mathrm{~nm}$ spectral radiance), averaged over 20 observations of the quiet Sun at disk center; the thick line is the flatness.

where $\boldsymbol{x}$ and $\boldsymbol{x}+\boldsymbol{\ell}$ go through all the field at a given $\|\boldsymbol{\ell}\|$. The normalized structure functions are:

$S_{\text {norm }}^{q}(\ell) \equiv S^{q}(\ell) /\left(S^{2}(\ell)\right)^{q / 2}$

$S_{\text {norm }}^{4}(\ell)$ is called the flatness, $F(\ell)$.

In phenomenological turbulence like Kolmogorov (1941) or Iroshnikov-Kraichnan (Iroshnikov 1963; Kraichnan 1965), the structure functions of the velocity are power laws of index $\zeta_{q}$ with $\zeta_{q} \propto q$, and this leads to normalized structure functions that are constant as a function of the scale $\ell$. When higher-order normalized structure functions, in particular the flatness, depend on $\ell$, it is a deviation from the above-mentioned classical phenomenologies of turbulence and the field is intermittent.

$\mathrm{S}$ VI $93.3 \mathrm{~nm}$ spectral radiance. The normalized structure functions for the scalar field of moment 1 (S VI $93.3 \mathrm{~nm}$ spectral radiance) averaged over 20 observations of the quiet Sun at disk center are shown in Fig. 12. They clearly rise when the spatial scales become smaller, especially when smaller than $15 \mathrm{Mm}$. This rise is characteristic of correlations in the field and of intermittency, and can also be seen in the normalized structure functions of the other radiance moments (of Ly $\varepsilon$ and S VI $94.4 \mathrm{~nm}$ ). However, when studying turbulent fields, the fields in which the intermittency is of primary interest are the magnetic and velocity fields. Consequently we now examine the latter.

S VI $93.3 \mathrm{~nm}$ line shift. When the field is a vector field $\boldsymbol{a}(\boldsymbol{x})$, it is usually projected longitudinally when computing the structure functions (in Eq. (3), $\delta_{\ell} a(\boldsymbol{x})$ is replaced by $\delta_{\ell} a(\boldsymbol{x}) \cdot \boldsymbol{\ell} / \ell$ ). In our case, as well as in magnetic field observations like Abramenko et al. (2002), we have no choice but to do this projection along the line-of-sight unitary vector $\boldsymbol{e}_{\text {los }}$ :

$S^{q}(\ell)=\left\langle\left|(\boldsymbol{v}(\boldsymbol{x}+\boldsymbol{\ell})-\boldsymbol{v}(\boldsymbol{x})) \cdot \boldsymbol{e}_{\operatorname{los}}\right|^{q}\right\rangle_{\boldsymbol{x},\|\boldsymbol{\ell}\|=\ell}$.

The resulting average normalized structure functions are shown in the top of Fig. 13. Because of the noise (which is not spatially correlated) in the fields of moment (2) and perhaps because of other effects discussed later, these structure functions are much flatter than in the case of moment (1), even if a small rise at the small scales can still be seen. However, when plotting the flatnesses of the field of moment (2) at all dates used to compute the
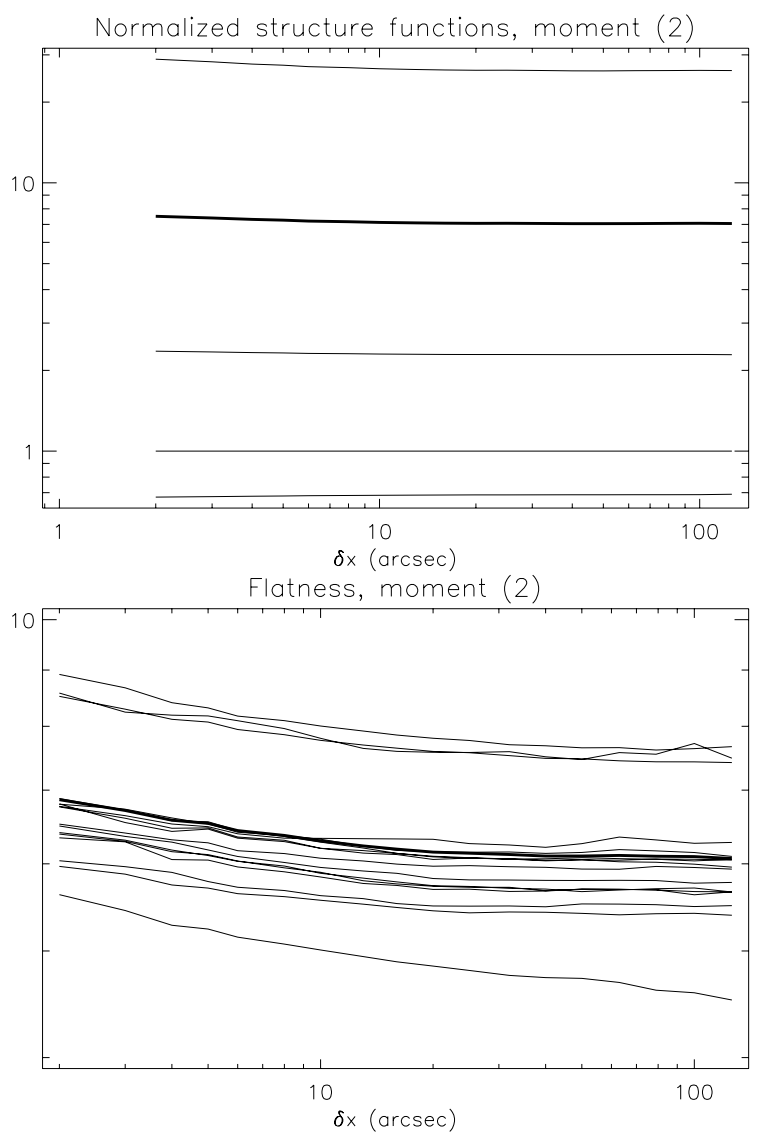

Fig. 13. Top: normalized structure functions of index 1 to 5 (bottom to top) for the field of moment (2) (S VI $93.3 \mathrm{~nm}$ line shift), averaged over 16 observations of the quiet Sun in the disk center; the thick line is the flatness. Bottom: flatness of the field of moment (2) at the dates used to compute the average (which is shown with the thick line).

average (Fig. 13 bottom), the rise of the flatness is systematic, and this gives a higher level of confidence in the rise observed on average. It thus seems that we can observe intermittency in the velocity field of the $\mathrm{S}$ VI $93.3 \mathrm{~nm}$ line.

\section{Discussion}

Many difficulties arise when attempting to compute the statistics presented here, mainly because of trade-offs between the need to obtain large fields at high spatial resolution over a limited time (during which the Sun has not changed much), and to:

- send all the required data to the ground with a given bandwidth (or to keep them temporarily in the limited SUMER memory);

- have long enough exposure times so as to have enough signal.

Because of the first trade-off we need to compute the moments of the lines on board and compress them by limiting their dynamics (see Appendix A), and the second trade-off is at the origin of the noise problem, which is particularly important for the line shift data (moment 2). But with the current instruments there is no other alternative: we think that the data set we used here is one of the most adapted to the purpose.

Despite the difficulties, the tentative results presented here support the presence in the transition region of small scales created by turbulence and the intermittent nature of turbulent fields. 
To our knowledge this is the first determination of the intermittency of the velocity field in an ultraviolet line. These small scales, at which dissipation is more efficient, may play a role in the heating of the plasma of the solar upper atmosphere, particularly of the corona (if confirmed by similar observations in hotter lines).

Improved observations should be obtained with more sensitive spectrographs, such as the ones from Solar B and Solar Orbiter, which will allow us to make progress by increasing spatial resolution while also increasing counting statistics and thus allow, in particular, a better determination of the velocity field. However, there will still be compromises to be made on the path to "ideal" observations which would have less noise, better spatial and temporal resolution, and which would include lines emitted at hotter temperatures further up in the corona, closer to where the heating is believed to take place. One step further could be attained by using novel ultraviolet imaging spectrograph designs, such as the MOSES spectroimager (Kankelborg \& Thomas 2001) or an Imaging UV Fourier Transform Spectrometer (Millard et al. 2004).

Acknowledgements. The authors acknowledge partial financial support from the PNST (Programme National Soleil-Terre) program of INSU (CNRS) and from European Union grant HPRN-CT-2001-00310 (TOSTISP network). They would like to thank Klaus Wilhelm and the anonymous referee for their valuable comments on this paper. The SUMER project is supported by DLR, CNES, NASA and the ESA PRODEX Programme (Swiss contribution). SoHO is a project of international cooperation between ESA and NASA.

\section{Appendix A: The compression algorithm}

The need to send the data of several full-Sun images (one per moment) to the ground at high resolution, in a limited time and with a limited bandwidth (10.5 kbps), forced us not only to compute the moments on board ("compression" method number 17 of the SUMER Operations Guide) but also to limit the number of bits used for one moment in one pixel. The principle of this second "compression" is to reduce the dynamics to an 8-bit unsigned byte and to saturate high (and low, in the case of line shifts) values.

To perform this, the function near is defined by the code corresponding to:

$\operatorname{near}(x)=\operatorname{byte}(\min (x+0.5,255))$

where $\min (\bullet, \bullet)$ returns the smallest of its both arguments and byte $(\bullet)$ transforms its argument into a one-byte integer lower approximate value.
If $M_{1}, \ldots, M_{5}$ are the 5 moments computed by the compression scheme number 17 for one pixel of the image, then the values transmitted to the ground are:

$M_{1}^{\prime}:=\operatorname{near}\left(255 M_{1} / C_{1}\right)$

$M_{2}^{\prime}:=\operatorname{near}\left(\max \left(10 M_{2}+128,0\right)\right)$

$M_{3}^{\prime}:=\operatorname{near}\left(10 M_{3}\right)$

$M_{4}^{\prime}:=\operatorname{near}\left(255 M_{4} / C_{2}\right)$

$M_{5}^{\prime}:=\operatorname{near}\left(255 M_{5} / C_{3}\right)$

where $C_{i}$ is the COMPAR $i$ parameter given in the headers of the FITS file.

The inverse operation is performed on the ground to recover approximations of the original values of $M_{1}, \ldots, M_{5}$.

On-board computation of the moments and further compression of the data were necessary to get the results presented in this paper from SoHO/SUMER data. However, future observations of this kind should learn from the limitations found here: even if they could still benefit from such a method, there is a need for more signal in the original spectral data, and for a compression scheme that allows better dynamics (like a lossless compression scheme applied to data with more than 8 bits).

\section{References}

Abramenko, V. I., Yurchyshyn, V. B., Wang, H., Spirock, T. J., \& Goode, P. R. 2002, ApJ, 577,487

Aletti, V., Velli, M., Bocchialini, K., et al. 2000, ApJ, 544, 550

Aschwanden, M. J., Tarbell, T. D., Nightingale, R. W., et al. 2000, ApJ, 535, 1047

Benz, A. O., Krucker, S., Acton, L. W., \& Bastian, T. S. 1997, A\&A, 320, 993

Berghmans, D., Clette, F., \& Moses, D. 1998, A\&A, 336, 1039

Brekke, P., Hassler, D. M., \& Wilhelm, K. 1997, Sol. Phys., 175, 349

Burlaga, L. F. 1991, Geophys. Res. Lett., 18, 1651

Chae, J., Yun, H. S., \& Poland, A. I. 1998, ApJS, 114, 151

Curdt, W., Brekke, P., Feldman, U., et al. 2001, A\&A, 375, 591

Gomez, D. O., Martens, P. C. H., \& Golub, L. 1993, ApJ, 405, 767

Hudson, H. S. 1991, Sol. Phys., 133, 357

Iroshnikov, P. 1963, Astron. Zh., 40, 742

Kankelborg, C. C., \& Thomas, R. J. 2001, in Proc. SPIE, 4498, UV/EUV and Visible Space Instrumentation for Astronomy and Solar Physics, O. H. Siegmund; S. Fineschi; M. A. Gummin; Eds., 16

Kolmogorov, A. 1941, Dokl. Akad. Nauk. SSSR, 30, 299

Kraichnan, R. 1965, Phys. Fluids, 8, 1385

Marsch, E. \& Tu, C.-Y. 1997, Nonlinear processes in Geophysics, 4, 101

Martens, P. C. H., \& Gomez, D. O. 1992, PASJ, 44, L187

Millard, A., Lemaire, P., \& Vial, J.-C. 2004, in 35th COSPAR Scientific Assembly, 1749

Parnell, C. E., \& Jupp, P. E. 2000, ApJ, 529, 554

Patsourakos, S., \& Vial, J.-C. 2002, A\&A, 385, 1073

Peter, H., \& Judge, P. G. 1999, ApJ, 522, 1148

Wilhelm, K., Axford, W., Curdt, W., et al. 1989, in The SOHO Mission (ESA-SP-1104), scientific and technical aspects of the instruments (ESA), 31

Wilhelm, K., Curdt, W., Marsch, E., et al. 1995, Sol. Phys., 162, 189 